OKHEP-98-09

\title{
Mode-by-mode summation for the zero point electromagnetic energy of an infinite cylinder
}

\author{
Kimball A. Milton* \\ Department of Physics and Astronomy, University of Oklahoma, Norman, Oklahoma 73019, USA
}

\author{
A. V. Nesterenko \\ Physical Department, Moscow State University, Moscow, 119 899, Russia \\ V. V. Nesterenko ${ }^{\dagger}$ \\ Bogoliubov Laboratory of Theoretical Physics, Joint Institute for Nuclear Research \\ Dubna, 141980, Russia
}

(October 11, 2018)

\begin{abstract}
Using the mode-by-mode summation technique the zero point energy of the electromagnetic field is calculated for the boundary conditions given on the surface of an infinite solid cylinder. It is assumed that the dielectric and magnetic characteristics of the material which makes up the cylinder $\left(\varepsilon_{1}, \mu_{1}\right)$ and of that which makes up the surroundings $\left(\varepsilon_{2}, \mu_{2}\right)$ obey the relation $\varepsilon_{1} \mu_{1}=$ $\varepsilon_{2} \mu_{2}$. With this assumption all the divergences cancel. The divergences are regulated by making use of zeta function techniques. Numerical calculations are carried out for a dilute dielectric-diamagnetic cylinder and for a perfectly conducting cylindrical shell. The Casimir energy in the first case vanishes, and in the second is in a complete agreement with that obtained by DeRaad and Milton who employed a Green's function technique with an ultraviolet regulator.
\end{abstract}

12.20.Ds, 03.70.+k,03.50.De,11.10.Lm

Typeset using REVTEX

*Electronic address: milton@mail.nhn.ou.edu

$\dagger$ Electronic address: nestr@thsun1.jinr.dubna.su 


\section{INTRODUCTION}

Calculation of the Casimir energy for nontrivial boundary conditions is a subject of intense ongoing activity. In spite of this, the experience accumulated in this area still does not allow one to predict, without involved calculation, even the sign of this energy [1,2]. In this connection the development of new effective methods of calculating the Casimir energy is doubtless of interest.

In recent papers [3, 4] the zero point energy of the electromagnetic field with boundary conditions given on a sphere has been calculated by a method of direct summation of the eigenfrequencies. A substantial point here was the use of contour integration in the complex frequency plane [5]. The divergences in this problem were removed by two subsequent steps. The first was accomplished by the subtraction of the vacuum energy of an infinite homogeneous space and the second made use of the technique of zeta function regularization. The same method was also applied for calculating the Casimir energy of a massless scalar field obeying Dirichlet and Neumann boundary conditions on a sphere [3]. The same technique has recently been used to rederive the electromagnetic Casimir energy for a spherical shell [6]. The possibility of incorporating in this approach the dielectric and magnetic properties of the media was demonstrated in [4. These calculations are distinguished by a certain concision and simplicity in comparison with the Green's function techniques [2], which, however, retain superiority with respect to physical interpretation.

The present paper seeks to show the efficiency of the mode summation method in calculating the zero point energy of an electromagnetic field when the boundary conditions are given on an infinite circular cylinder. When applying the Green's function techniques [2, 7, 8], this problem turns out to be more complicated than the corresponding calculation of the Casimir energy for sphere [9 15].

The layout of the paper is as follows. In Sec. II the general integral representation is derived for the Casimir energy of an infinite solid cylinder surrounded by an uniform medium. The permittivity and permeability of the cylinder material $\left(\varepsilon_{1}, \mu_{1}\right)$ and those of the surroundings $\left(\varepsilon_{2}, \mu_{2}\right)$ are considered to be arbitrary. In principle they may depend on the frequency of the electromagnetic oscillations (dispersion of the media), but this point is beyond the scope of the present paper. In Sec. III it is assumed that the electromagnetic characteristics obey the condition $\varepsilon_{1} \mu_{1}=\varepsilon_{2} \mu_{2}=c^{-2}$ where $c$ is the speed of the light in the media (in units of that in the vacuum). When this condition is satisfied, all the divergences cancel between interior and exterior modes. Those divergences are regulated by employing the zeta function technique. In Sec. IV the cases when $\xi^{2} \ll 1$ and $\xi^{2}=1$ are considered numerically, $\xi^{2}$ being $\left(\varepsilon_{1}-\varepsilon_{2}\right)^{2} /\left(\varepsilon_{1}+\varepsilon_{2}\right)^{2}$. The first case gives the Casimir energy of a dilute dielectric-diamagnetic cylinder, while the second case corresponds to a perfectly conducting infinitely thin cylindrical shell. Remarkably, the Casimir energy obtained for a tenuous medium vanishes, as it does for a tenuous dielectric cylinder. The result obtained in the second case is identical to that obtained by the Green's function method of calculating the energy and regulating the divergences by use of an ultraviolet regulator [2]. In Sec. V (Conclusion) the significance of the universal results obtained are discussed. 


\section{INTEGRAL REPRESENTATION FOR THE CASIMIR ENERGY}

We shall consider the following configuration. An infinite cylinder of radius $a$ is placed in an uniform unbounded medium. The permittivity and the permeability of the material making up the cylinder are $\varepsilon_{1}$ and $\mu_{1}$, respectively, and those for surrounding medium are $\varepsilon_{2}$ and $\mu_{2}$. It is assumed that the conductivity in both the media is zero. We will compute the Casimir energy per unit length of the cylinder.

In the mode summation method, the renormalized Casimir energy is defined by

$$
E=\frac{1}{2} \sum_{\{p\}}\left(\omega_{p}-\bar{\omega}_{p}\right),
$$

where $\omega_{p}$ are the classical eigenfrequencies of the electromagnetic oscillations in the system under consideration, and $\bar{\omega}_{p}$ are those in the absence of any boundary, that is, when either medium fills all space. (When the precise meaning is not required, we denote this by the formal limit $a \rightarrow \infty$.) The set $\{p\}$ stands for a complete set of quantum numbers (discrete and continuous) which is determined by the symmetry of the problem. Either sum in Eq. (2.1) diverges, therefore a preliminary regularization is required.

In order for the eigenfrequencies to be found one needs to solve Maxwell's equations for the given configuration with allowance for the appropriate boundary conditions on the lateral surface of the cylinder. As is well known, it is sufficient to require the continuity of the tangential components of the electric field $\mathbf{E}$ and of the magnetic field $\mathbf{H}$ [16]. In terms of the cylindrical coordinates $(r, \theta, z)$ the eigenfunctions of the given boundary value problem contain the multiplier

$$
\exp \left(-i \omega t+i k_{z} z+i n \theta\right)
$$

and their dependence on $r$ is described by the cylindrical Bessel functions $J_{n}$ for $r<a$ and by the Hankel functions of the first kind $H_{n} \equiv H_{n}^{(1)}$ for $r>a$. The eigenfrequencies are the roots of the equation (Ref. [16], p. 526)

$$
f_{n}\left(k_{z}, \omega, a\right)=0
$$

where

$$
f_{n} \equiv \lambda_{1}^{2} \lambda_{2}^{2} \Delta_{n}^{\mathrm{TE}}\left(\lambda_{1} a, \lambda_{2} a\right) \Delta_{n}^{\mathrm{TM}}\left(\lambda_{1} a, \lambda_{2} a\right)-n^{2} \omega^{2} k_{z}^{2}\left(\varepsilon_{1} \mu_{1}-\varepsilon_{2} \mu_{2}\right)^{2}\left(J_{n}\left(\lambda_{1} a\right) H_{n}\left(\lambda_{2} a\right)\right)^{2},
$$

with

$$
\begin{array}{r}
\Delta_{n}^{\mathrm{TE}}\left(\lambda_{1} a, \lambda_{2} a\right)=a \mu_{1} \lambda_{2} J_{n}^{\prime}\left(\lambda_{1} a\right) H_{n}\left(\lambda_{2} a\right)-a \mu_{2} \lambda_{1} J_{n}\left(\lambda_{1} a\right) H_{n}^{\prime}\left(\lambda_{2} a\right), \\
\Delta_{n}^{\mathrm{TM}}\left(\lambda_{1} a, \lambda_{2} a\right)=a \varepsilon_{1} \lambda_{2} J_{n}^{\prime}\left(\lambda_{1} a\right) H_{n}\left(\lambda_{2} a\right)-a \varepsilon_{2} \lambda_{1} J_{n}\left(\lambda_{1} a\right) H_{n}^{\prime}\left(\lambda_{2} a\right), \\
\lambda_{i}^{2}=k_{i}^{2}-k_{z}^{2}, \quad k_{i}^{2}=\varepsilon_{i} \mu_{i} \omega^{2}, \quad i=1,2, \quad n=0, \pm 1, \pm 2, \ldots
\end{array}
$$

The indices TE and TM will be explained below. The prime on the functions $J_{n}$ and $H_{n}$ means differentiation with respect to their arguments. For given $k_{z}$ and $n$ Eq. (2.3) has an 
infinite sequence of roots $\omega_{n m}\left(k_{z}\right), m=1,2, \ldots$, these frequencies being the same inside and outside the cylinder [17]. In view of this the Casimir energy (2.1) can be rewritten as

$$
E=\frac{1}{2} \int_{-\infty}^{\infty} \frac{d k_{z}}{2 \pi} \sum_{n=-\infty}^{\infty} \sum_{m=1}^{\infty}\left[\omega_{n m}\left(k_{z}\right)-\bar{\omega}_{n m}\left(k_{z}\right)\right],
$$

where $\bar{\omega}_{n m}\left(k_{z}\right)$ stands for the uniform medium subtraction referred to above.

The next step in our consideration is a representation of the sum in Eq. (2.6) in terms of the contour integral [5]

$$
E=\frac{1}{2} \int_{-\infty}^{\infty} \frac{d k_{z}}{2 \pi} \sum_{n=-\infty}^{\infty} \frac{1}{2 \pi i} \frac{1}{2} \oint_{C} \omega d_{\omega} \ln \frac{f_{n}\left(k_{z}, \omega, a\right)}{f_{n}\left(k_{z}, \omega, \infty\right)}
$$

Integration in (2.7) is conducted along a closed path $C$ in the complex $\omega$ plane which consists of two parts: $C_{+}$which encloses the positive roots of Eq. (2.3) in a counterclockwise sense, and $C_{-}$which encircles the negative roots in a clockwise sense. Therefore, we face to task of investigating the properties of the function $f_{n}\left(k_{z}, \omega, a\right)$ specifying the frequency equation. (However, the result (2.7) may be easily shown to be equivalent to the corresponding Green's function formulation. See Appendix A.) Generally this is a problem of extreme difficulty. Therefore, in the next sections we shall consider specific cases introducing simplifying assumptions.

The method of calculation of the Casimir energy proposed above can straightforward be generalized to the dispersive media. To this end, it is sufficient to treat the parameters $\varepsilon_{i}$ and $\mu_{i}, i=1,2$ in the frequency equation (2.3) as given functions of the frequency $\omega$. However, this issue is beyond the scope of the present paper.

\section{CASIMIR ENERGY OF AN INFINITE CYLINDER WHEN $\varepsilon_{1} \mu_{1}=\varepsilon_{2} \mu_{2}$}

We assume that the permittivity and permeability of the cylinder material $\left(\varepsilon, \mu_{1}\right)$ and of the surroundings $\left(\varepsilon_{2}, \mu_{2}\right)$ are not arbitrary but satisfy the condition

$$
\varepsilon_{1} \mu_{1}=\varepsilon_{2} \mu_{2}=c^{-2}
$$

where $c$ is the light speed in either medium (in units of the speed of light in vacuum). The physical implications of this condition can be found in 18 20. When equation (3.1) holds, we have $\lambda_{1}=\lambda_{2}=\lambda$, and the frequency equation (2.3) is simplified considerably. It breaks down into two equations: for the transverse-electric (TE) oscillations

$$
\Delta_{n}^{\mathrm{TE}}(\lambda, a) \equiv \lambda a\left[\mu_{1} J_{n}^{\prime}(\lambda a) H_{n}(\lambda a)-\mu_{2} J_{n}(\lambda a) H_{n}^{\prime}(\lambda a)\right]=0
$$

and for the transverse-magnetic (TM) oscillations

$$
\Delta_{n}^{\mathrm{TM}}(\lambda, a) \equiv \lambda a\left[\varepsilon_{1} J_{n}^{\prime}(\lambda a) H_{n}(\lambda a)-\varepsilon_{2} J_{n}(\lambda a) H_{n}^{\prime}(\lambda a)\right]=0 .
$$

In the general case [see Eq. (2.4)] such a decomposition occurs only for oscillations with $n=0$. In Eqs. (3.2) and (3.3) $\lambda$ is the eigenvalue of the corresponding transverse [membranelike] boundary value problem [21] 


$$
\lambda^{2}=\frac{\omega^{2}}{c^{2}}-k_{z}^{2}
$$

Classification of the solutions of Maxwell's equations without sources in terms of the TE- and TM-modes originates in waveguide theory [16,21,22]. The main distinction of the propagation of electromagnetic waves in waveguides, in contrast to the same process in unbounded space, is that a purely transverse wave cannot propagate in a waveguide. The wave in waveguide must necessarily contain either longitudinal electric or magnetic fields. The first case is referred to as the waves of electric type [transverse-magnetic (TM) waves] and in the second case one is dealing with waves of magnetic type [or transverse-electric (TE) waves]. This classification proves to be convenient in studies of electromagnetic oscillations in closed resonators as well.

Replacing the function $f_{n}\left(k_{z}, \omega, a\right)$ in Eq. (2.7) by the left hand sides of Eq. (3.2) and (3.3) and changing to the integration variable $\lambda$ we arrive at the following representation for the Casimir energy

$$
E=-\frac{c}{2} \int_{-\infty}^{\infty} \frac{d k_{z}}{2 \pi} \sum_{n=-\infty}^{\infty} \frac{1}{2 \pi i} \frac{1}{2} \oint_{C^{\prime}} \sqrt{\lambda^{2}+k_{z}^{2}} d_{\lambda} \ln \frac{\Delta_{n}^{\mathrm{TE}}(\lambda a) \Delta_{n}^{\mathrm{TM}}(\lambda a)}{\Delta_{n}^{\mathrm{TE}}(\infty) \Delta_{n}^{\mathrm{TM}}(\infty)}
$$

Here we have distorted the contour of integration to $C^{\prime}=C_{+}^{\prime}+C_{-}^{\prime}$. We take $C_{+}^{\prime}$ to consist of a straight line parallel to, and just to the right of, the imaginary axis $(-i \infty,+i \infty)$ closed by a semicircle of an infinitely large radius in the right half-plane. $C_{-}^{\prime}$ similarly is a line parallel to, and just to the left of, the imaginary axis, closed by an infinite semicircle in the left hand plane. On both semicircles the argument of the logarithm function in Eq. (3.5) tends to 1 . As a result these parts of the contour $C^{\prime}$ do not give any contribution to the Casimir energy $E$. When integrating along the imaginary axis we chose the branch line of the function $\varphi(\lambda)=\sqrt{\lambda^{2}+k_{z}^{2}}$ to run between $-i k_{z}$ and $i k_{z}$, where $k_{z}=+\sqrt{k_{z}^{2}}>0$. In terms of $y=\operatorname{Im} \lambda$ we have

$$
\varphi(i y)= \begin{cases}i \sqrt{y^{2}-k_{z}^{2}}, & y>k_{z} \\ \pm \sqrt{k_{z}^{2}-y^{2}}, & |y|<k_{z} \\ -i \sqrt{y^{2}-k_{z}^{2}}, & y<-k_{z}\end{cases}
$$

where the sign on the middle form depends on whether we are to the right or the left of the cut. Thus contributions to Eq. (3.5) due to the integration along the segment of the imaginary axis $\left(-i k_{z}, i k_{z}\right)$ cancel between $C_{+}^{\prime}$ and $C_{-}^{\prime}$, and Eq. (3.5) acquires the form

$$
E=-\frac{c}{2 \pi^{2}} \sum_{n=-\infty}^{\infty} \int_{0}^{\infty} d k_{z} \int_{k_{z}}^{\infty} \sqrt{y^{2}-k_{z}^{2}} d_{y} \ln \frac{\Delta_{n}^{\mathrm{TE}}(i a y) \Delta_{n}^{\mathrm{TM}}(i a y)}{\Delta_{n}^{\mathrm{TE}}(i \infty) \Delta_{n}^{\mathrm{TM}}(i \infty)} .
$$

Changing the order of integration of $k_{z}$ and $y$ and taking into account the value of the integral

$$
\int_{0}^{y} d k_{z} \sqrt{y^{2}-k_{z}^{2}}=\frac{\pi}{4} y^{2}
$$

we obtain after substitution $a y \rightarrow y$ 


$$
E=-\frac{c}{8 \pi a^{2}} \sum_{n=-\infty}^{\infty} \int_{0}^{\infty} y^{2} d_{y} \ln \frac{\Delta_{n}^{\mathrm{TE}}(i y) \Delta_{n}^{\mathrm{TM}}(i y)}{\Delta_{n}^{\mathrm{TE}}(i \infty) \Delta_{n}^{\mathrm{TM}}(i \infty)} .
$$

Further we shall need the modified Bessel functions $I_{n}(y)$ and $K_{n}(y)$ [23]

$$
\begin{aligned}
I_{n}(y) & =i^{-n} J_{n}(i y) \\
K_{n}(y) & =i^{n+1} \frac{\pi}{2} H_{n}(i y)
\end{aligned}
$$

and their asymptotics at fixed $n$ and $y \rightarrow \infty$

$$
\begin{aligned}
I_{n}(y) & \simeq \frac{e^{y}}{\sqrt{2 \pi y}}, \quad I_{n}^{\prime}(y) \simeq \frac{e^{y}}{\sqrt{2 \pi y}}, \\
K_{n}(y) & \simeq \sqrt{\frac{\pi}{2 y}} e^{-y}, \quad K_{n}^{\prime}(y) \simeq-\sqrt{\frac{\pi}{2 y}} e^{-y} .
\end{aligned}
$$

With the help of this we derive from Eqs. (3.2) and (3.3)

$$
\begin{gathered}
\frac{\Delta_{n}^{\mathrm{TE}}(i y)}{\Delta_{n}^{\mathrm{TE}}(i \infty)}=\frac{2 y}{\mu_{1}+\mu_{2}}\left[\mu_{1} I_{n}^{\prime}(y) K_{n}(y)-\mu_{2} I_{n}(y) K_{n}^{\prime}(y)\right], \\
\frac{\Delta_{n}^{\mathrm{TM}}(i y)}{\Delta_{n}^{\mathrm{TM}}(i \infty)}=\frac{2 y}{\varepsilon_{1}+\varepsilon_{2}}\left[\varepsilon_{1} I_{n}^{\prime}(y) K_{n}(y)-\varepsilon_{2} I_{n}(y) K_{n}^{\prime}(y)\right] .
\end{gathered}
$$

Making use of all this, we can recast Eq. (3.9) into the form

$$
\begin{aligned}
E= & \frac{c}{4 \pi a^{2}} \sum_{n=-\infty}^{\infty} \int_{0}^{\infty} y d y \ln \left\{\frac { 4 y ^ { 2 } } { \varepsilon + \varepsilon ^ { - 1 } + 2 } \left[\left(I_{n}^{\prime}(y) K_{n}(y)\right)^{2}+\left(I_{n}(y) K_{n}^{\prime}(y)\right)^{2}\right.\right. \\
& \left.\left.-\left(\varepsilon+\varepsilon^{-1}\right) I_{n}(y) I_{n}^{\prime}(y) K_{n}(y) K_{n}^{\prime}(y)\right]\right\} .
\end{aligned}
$$

Here a new notation $\varepsilon=\varepsilon_{1} / \varepsilon_{2}$ has been introduced, $\mu$ has been eliminated by condition (3.1), and when going from (3.9) to (3.15) an integration by parts has been done, the boundary terms being omitted. The last point will be justified further when the removal of the divergences is discussed. The argument of the logarithm in Eq. (3.15) is simplified considerably if one uses the value of the Wronskian of the modified Bessel functions $I_{n}(y)$ and $K_{n}(y)$ [23]

$$
I_{n}(y) K_{n}^{\prime}(y)-I_{n}^{\prime}(y) K_{n}(y)=-\frac{1}{y}
$$

and the identity

$$
I_{n}(y) K_{n}^{\prime}(y)+I_{n}^{\prime}(y) K_{n}(y)=\left(I_{n}(y) K_{n}(y)\right)^{\prime} .
$$

Finally Eq. (3.15) acquires the form 


$$
E=\sum_{n=-\infty}^{\infty} E_{n}
$$

where

$$
E_{n}=\frac{c}{4 \pi a^{2}} \int_{0}^{\infty} y d y \ln \left\{1-\xi^{2}\left[y\left(I_{n}(y) K_{n}(y)\right)^{\prime}\right]^{2}\right\}
$$

with $\xi=(1-\varepsilon) /(1+\varepsilon)$. This is a simple unregulated generalization of (4.5) of Ref. [2], and the cylindrical analog of the spherical form (7.1) of Ref. [12].

From the asymptotics (3.12) and (3.13) it follows that the integral in (3.19) diverges logarithmically when $y \rightarrow \infty$. At the same time the sum over $n$ in Eq. (3.18) also diverges because at large $n$ the uniform asymptotic expansion of the modified Bessel functions gives [23] (see (4.6) of [2])

$$
\left.E_{n}\right|_{n \rightarrow \infty} \simeq-\frac{c \xi^{2}}{16 \pi a^{2}} \int_{0}^{\infty} \frac{z^{5} d z}{\left(1+z^{2}\right)^{3}} \equiv E^{\infty}
$$

Here the change of variables $y=n z$ has been done. Disregarding for the moment that the integral in Eq. (3.20) is divergent, we employ here the Riemann zeta function technique 24,25] for attributing a finite value to the sum in (3.18)

$$
\begin{aligned}
E & =\sum_{n=-\infty}^{\infty}\left(E_{n}-E^{\infty}+E^{\infty}\right) \\
& =\sum_{n=-\infty}^{\infty}\left(E_{n}-E^{\infty}\right)+\sum_{n=-\infty}^{\infty} E^{\infty} \\
& =\sum_{n=-\infty}^{\infty} \bar{E}_{n}+E^{\infty} \sum_{n=-\infty}^{\infty} n^{0},
\end{aligned}
$$

where $\bar{E}_{n}$ stands for the "renormalized" partial Casimir energy

$$
\bar{E}_{n}=E_{n}-E^{\infty}, \quad n=0, \pm 1, \ldots
$$

We now have to treat the product of two divergent expressions $E^{\infty} \cdot \sum_{n=-\infty}^{\infty} n^{0}$ more precisely, by presenting it in the following form

$$
\begin{aligned}
E^{\infty} \cdot \sum_{n=-\infty}^{\infty} n^{0} & =-\frac{c \xi^{2}}{16 \pi a^{2}} \lim _{s \rightarrow 0^{+}} \int_{0}^{\infty} \frac{z^{5-s} d z}{\left(1+z^{2}\right)^{3}} \cdot[2 \zeta(s)+1] \\
& =-\frac{c \xi^{2}}{16 \pi a^{2}} \lim _{s \rightarrow 0^{+}}\left(\frac{1}{s}-\frac{3}{4}\right) \cdot\left[2 \zeta^{\prime}(s) s\right] \\
& =-\frac{c \xi^{2}}{16 \pi a^{2}} \lim _{s \rightarrow 0^{+}}\left(\frac{1}{s}-\frac{3}{4}\right) \cdot[-\ln (2 \pi) s] \\
& =\frac{c \xi^{2}}{16 \pi a^{2}} \ln (2 \pi) .
\end{aligned}
$$

Finally, the Casimir energy acquires the form 


$$
E=\sum_{n=-\infty}^{\infty} \bar{E}_{n}+\frac{c \xi^{2}}{16 \pi a^{2}} \ln (2 \pi)
$$

Now we deduce from Eqs. (3.22), (3.19), and (3.20) for $\bar{E}_{n}$

$$
\begin{gathered}
\bar{E}_{n}=\frac{c}{4 \pi a^{2}}\left\{\int_{0}^{\infty} y d y \ln \left[1-\xi^{2} \sigma_{n}^{2}(y)\right]+\frac{\xi^{2}}{4} \int_{0}^{\infty} \frac{z^{5} d z}{\left(1+z^{2}\right)^{3}}\right\}, \\
\bar{E}_{-n}=\bar{E}_{n}, \quad n=0,1,2, \ldots,
\end{gathered}
$$

where $\sigma_{n}(y)=y\left(I_{n}(y) K_{n}(y)\right)^{\prime}$. Since both integrals in Eq. (3.25) rdiverge, the finite sum is to interpreted in a precise manner as specified below.

The removal of the divergences by making use of the $\zeta$ function justifies dropping the boundary terms in the integration by parts when we went from Eq. (3.9) to (3.15). To see this one can at first remove the divergences in Eq. (3.9) by employing the Riemann $\zeta$ function as described above. After that the integration by parts in the formula for the renormalized partial Casimir energy $\bar{E}_{n}$ can be done, with boundary terms now vanishing rigorously. Proceeding in that way one again arrives at Eqs. (3.25) and (3.26).

In order for Eq. (3.25) to be cast in a form suitable for numerical evaluations both terms there should be placed under s single integral sign. To this end, for $n \neq 0$ the change of variables $z=y / n$ has to be done in the second term. This change is inverse to the one which has led to the asymptotic form (3.20). This yields

$$
\bar{E}_{n}=\frac{c}{4 \pi a^{2}} \int_{0}^{\infty} y d y\left\{\ln \left[1-\xi^{2} \sigma_{n}^{2}(y)\right]+\frac{\xi^{2}}{4} \frac{y^{4}}{\left(n^{2}+y^{2}\right)^{3}}\right\}, \quad n=1,2, \ldots
$$

For $n=0 \mathrm{Eq}$. (3.25) is rewritten as

$$
\bar{E}_{0}=\frac{c}{4 \pi a^{2}} \int_{0}^{\infty} y d y\left\{\ln \left[1-\xi^{2} \sigma_{0}^{2}(y)\right]+\frac{\xi^{2}}{4} \frac{y^{4}}{\left(1+y^{2}\right)^{3}}\right\} .
$$

The integrals in these formulæ converge because for $y \rightarrow 0$ and $n \neq 0$ we have [23]

$$
I_{n}(y) K_{n}(y) \rightarrow \frac{1}{2 n} .
$$

In this limit

$$
\sigma_{0}^{2}(y) \rightarrow 1
$$

On the other hand, for large $y$

$$
\sigma_{n}^{2}(y) \rightarrow \frac{1}{4 y^{2}}
$$

By making use of uniform asymptotics of the Bessel functions [23] we deduce from (3.27) 


$$
\left.\bar{E}_{n}\right|_{n \rightarrow \infty} \sim \frac{c \xi^{2}}{4 \pi a^{2}}\left[\frac{10-3 \xi^{2}}{960 n^{2}}-\frac{28224-7344 \xi^{2}+720 \xi^{4}}{15482880 n^{4}}+O\left(\frac{1}{n^{6}}\right)\right] .
$$

Thus the Casimir energy

$$
E=\bar{E}_{0}+2 \sum_{n=1}^{\infty} \bar{E}_{n}+\frac{c \xi^{2}}{16 \pi a^{2}} \ln (2 \pi)
$$

with $\bar{E}_{0}$ and $\bar{E}_{n}, n \geq 1$, given in (3.28) and (3.27), respectively, is finite 26]. One can advance further only by considering special cases and applying numerical calculations.

\section{DILUTE COMPACT CYLINDER AND PERFECTLY CONDUCTING CYLINDRICAL SHELL}

We begin by addressing the case when $\xi^{2} \ll 1$. Because we are assuming the condition $\varepsilon_{1} \mu_{1}=\varepsilon_{2} \mu_{1}=c^{-2}$ and $\xi^{2} \ll 1$, this is not the same situation as a dilute compact cylinder with $\left|\varepsilon_{1}-\varepsilon_{2}\right| \ll 1$ and $\mu_{1}=\mu_{2}=1$. In this case

$$
\xi^{2}=\left(\frac{\varepsilon_{1}-\varepsilon_{2}}{\varepsilon_{1}+\varepsilon_{2}}\right)^{2}=\frac{\left(\varepsilon_{1}-\varepsilon_{2}\right)^{2}}{4 \varepsilon^{2}},
$$

where $\varepsilon=\left(\varepsilon_{1}+\varepsilon_{2}\right) / 2$. Retaining in Eq. (3.28) only the terms proportional to $\xi^{2}$ we obtain

$$
\begin{aligned}
\bar{E}_{0} & \simeq \frac{c \xi^{2}}{4 \pi a^{2}} \int_{0}^{\infty} y d y\left[\frac{y^{4}}{4\left(1+y^{2}\right)^{3}}-\sigma_{0}^{2}(y)\right] \\
& \simeq \frac{c \xi^{2}}{4 \pi a^{2}}(-0.490878) .
\end{aligned}
$$

To estimate $\bar{E}_{n}, n>0$, we can use the leading asymptotic behavior (3.32)

$$
\bar{E}_{n} \sim \frac{c \xi^{2}}{4 \pi a^{2}}\left(\frac{1}{96 n^{2}}-\frac{7}{3840 n^{4}}\right)
$$

To a precision of $10^{-6}$, we evaluate Eq. (3.33) by substituting in the value of $\bar{E}_{0}$, (4.2), integrating $\bar{E}_{n}$ numerically for $n=1, \ldots 5$, and asymptotically using Eq. (3.32) for $n \geq 6$, with the result!

$$
\begin{aligned}
E & \simeq \frac{c \xi^{2}}{4 \pi a^{2}}\left(-0.490878+2 \sum_{n=1}^{5} \bar{E}_{n}+\frac{1}{48} \sum_{n=6}^{\infty} \frac{1}{n^{2}}-\frac{7}{1920} \sum_{n=6}^{\infty} \frac{1}{n^{4}}+\frac{1}{4} \ln (2 \pi)\right) \\
& =\frac{c \xi^{2}}{4 \pi a^{2}}(-0.490878+0.027638+0.003778-0.000007+0.459469) \\
& =\frac{c \xi^{2}}{4 \pi a^{2}}(0.000000) .
\end{aligned}
$$

\footnotetext{
${ }^{1}$ The cancellations here are very severe. If the asymptotic approximation were used for all $n$, a positive result would be found, $E \sim\left(c \xi^{2} / 4 \pi a^{2}\right)(-0.00108)$. Unlike for the spherical case, doing the integral exactly is essential.
} 
Thus the Casimir energy of a cylinder possessing the same speed of light inside and outside proves to be zero! This is to be contrasted with the positive Casimir energy found for a dilute ball with the same property [14, 12, 14,

$$
E_{\text {ball }} \approx \frac{3}{64 a} \xi^{2}=0.046875 \frac{\xi^{2}}{a} .
$$

It is further remarkable that the same zero result is found for a dilute dielectric cylinder, that is, one with $\mu=1$ everywhere and $\varepsilon>1$ inside the cylinder, and $\varepsilon=1$ outside, a result which may be most easily confirmed by summing the intermolecular van der Waals energies. That calculation is given in Appendix B. However, zero is not the universal value of the Casimir energy for cylinders, as we now remind the reader.

Of particular interest is the case when $\xi^{2}=1$. With $c=1$ in our formulas it corresponds to infinitely thin and perfectly conducting cylindrical shell (see Appendix C). Setting $\xi=1$ and $c=1$ in Eq. (3.28) we obtain by numerical integrationf

$$
\bar{E}_{0}=\frac{1}{4 \pi a^{2}}(-0.6517)=-0.05186 \frac{1}{a^{2}} .
$$

The sum $2 \sum_{n=1}^{\infty} \bar{E}_{n}$ in Eq. (3.33) can be found by making use of the two leading terms in the uniform asymptotic expansion

$$
\begin{aligned}
2 \sum_{n=1}^{\infty} \bar{E}_{n} & \simeq \frac{1}{4 \pi a^{2}}\left(\frac{7}{480} \sum_{n=1}^{\infty} \frac{1}{n^{2}}-\frac{5}{1792} \sum_{n=1}^{\infty} \frac{1}{n^{4}}\right)=\frac{1}{4 \pi a^{2}}\left(\frac{7}{480} \frac{\pi^{2}}{6}-\frac{5}{1792} \frac{\pi^{4}}{90}\right) \\
& =\frac{1}{4 \pi a^{2}} 0.0210=0.0018 \frac{1}{a^{2}} .
\end{aligned}
$$

With higher accuracy (up to $10^{-5}$ ) this sum has been calculated in [2] by integration of [3.27)]

$$
2 \sum_{n=1}^{\infty} \bar{E}_{n} \simeq \frac{1}{4 \pi a^{2}} \frac{1}{2} 0.0437=\frac{1}{4 \pi a^{2}} 0.0218=0.00174 \frac{1}{a^{2}} .
$$

Substituting Eqs. (4.6) and (4.8) into (3.33) we obtain for the Casimir energy of a perfectly conducting cylindrical shell

$$
E_{\text {shell }}=\frac{1}{4 \pi a^{2}}(-0.1704)=-0.01356 \frac{1}{a^{2}} .
$$

This is exactly the result first obtained by DeRaad and Milton [2,8]. It is worth noting here that unlike in that approach the use of the $\zeta$ function techniques enables us to dispense with introduction of the high-frequency cutoff function, although the latter is undoubtedly more physical.

While this paper was being completed the authors became aware of Ref. [27] where the vacuum energy of an infinite perfectly conducting cylindrical surface has also been rederived, to much higher accuracy, but using a rather more elaborate method.

\footnotetext{
${ }^{2}$ In the notation of Ref. [2] this is $-\frac{1}{8 \pi a^{2}}\left(S+R_{0}+\frac{1}{2} \ln 2 \pi\right)$-see Eqs. (5.5) and (5.6) of Ref. [2]. The $\ln 2 \pi$ term is cancelled by that in Eq. (3.33) here.

${ }^{3}$ This is exactly the same as $-\frac{1}{8 \pi a^{2}} R$ given by Eq. (5.11) of Ref. [2].
} 


\section{CONCLUSION}

When calculating the Casimir energy there appear to be many arbitrary methods of controlling and removing divergences. It is therefore reassuring that unique results emerge whatever regularization scheme is adopted. This is somewhat less trivial for this cylindrical case than for the case of a sphere, because of the subtleties associated with even space dimensions 28]. However, this universality seems to be characteristic of Casimir calculations even in cases where finiteness is not achieved, as for a dielectric ball without the condition (3.1) imposed [29,30]. Thus our understanding of the Casimir effect seems to be improving. However, this comforting conclusion must be tempered by the surprising new result that for both dilute dielectric-diamagnetic (satisfying $\varepsilon \mu=$ const.) cylinders, and for dilute dielectric cylinders, the Casimir energy vanishes. We have as yet no theoretical understanding of these zeroes.

\section{ACKNOWLEDGMENTS}

We thank M. Bordag for organizing the Fourth Workshop on Quantum Field Theory Under the Influence of External Conditions, which enabled the collaboration to take place. We are particularly grateful to A. Romeo for pointing out that the dielectric cylinder had vanishing Casimir energy, and thereby triggering the detection of a transcription error in the calculation of the energy for a dilute dielectric-diamagnetic cylinder. This work was accomplished with partial financial support of the Russian Foundation of Fundamental Research (Grant No. 97-01-00745) and of the U.S. Department of Energy (Grant No. DE-FG-0398ER41066).

\section{APPENDIX A: RELATION OF METHOD TO GREEN'S FUNCTION APPROACH}

Here we sketch the relation of the formula (2.7) to the Green's function formalism. We will confine our remarks to the case of a massless scalar field, as the generalization to, say, electromagnetism is rather immediate. We take the scalar Green's function to satisfy the differential equation

$$
\left(\frac{\partial^{2}}{\partial t^{2}}-\nabla^{2}\right) G\left(x, x^{\prime}\right)=-\delta\left(x-x^{\prime}\right),
$$

subject to appropriate boundary conditions. The stress tensor (use of the conformal stress tensor has the same effect) is

$$
t^{\mu \nu}=\partial^{\mu} \phi \partial^{\nu} \phi-\frac{1}{2} g^{\mu \nu} \partial_{\lambda} \phi \partial^{\lambda} \phi
$$

so, since the Green's function is given as a vacuum expectation value of a time-ordered product of fields,

$$
G\left(x, x^{\prime}\right)=-i\left\langle T \phi(x) \phi\left(x^{\prime}\right)\right\rangle
$$


the energy density is

$$
u=\left\langle t^{00}\right\rangle=-\left.\frac{i}{2}\left[\partial^{0} \partial^{0 \prime}+\nabla \cdot \nabla^{\prime}\right] G\left(x, x^{\prime}\right)\right|_{x=x^{\prime}} .
$$

To compute the total energy, we integrate $u$ over all space; then we can integrate by parts on one of the gradient terms, and use the differential equation (A1), omitting the delta function because the limit of point coincidence is understood:

$$
\nabla \cdot \nabla^{\prime} \rightarrow-\nabla^{2} \rightarrow-\frac{\partial^{2}}{\partial t^{2}}
$$

(The net effect is that the Lagrangian term in $t^{\mu \nu}$ does not contribute.) In terms of the Fourier transform of the Green's function

$$
G\left(x, x^{\prime}\right)=\int \frac{d \omega}{2 \pi} e^{-i \omega\left(t-t^{\prime}\right)} \mathcal{G}_{\omega}\left(\mathbf{r}, \mathbf{r}^{\prime}\right)
$$

the Casimir energy is

$$
E=-\left.i \int \frac{d \omega}{2 \pi} e^{-i \omega\left(t-t^{\prime}\right)} \int(d \mathbf{r}) \omega^{2} \mathcal{G}_{\omega}\left(\mathbf{r}, \mathbf{r}^{\prime}\right)\right|_{x=x^{\prime}}
$$

Now introduce eigenfunctions of the differential operator $\nabla^{2}$ subject to the same boundary conditions as $\mathcal{G}_{\omega}$ :

$$
\begin{aligned}
\nabla^{2} \psi(\mathbf{r}) & =-k_{p}^{2} \psi(\mathbf{r}), \\
\sum_{p} \psi_{p}(\mathbf{r}) \psi_{p}^{*}\left(\mathbf{r}^{\prime}\right) & =\delta\left(\mathbf{r}-\mathbf{r}^{\prime}\right), \\
\int(d \mathbf{r}) \psi_{p}^{*}(\mathbf{r}) \psi_{p^{\prime}}(\mathbf{r}) & =\delta_{p p^{\prime}}
\end{aligned}
$$

Then the Green's function has an eigenfunction expansion

$$
\mathcal{G}_{\omega}\left(\mathbf{r}, \mathbf{r}^{\prime}\right)=\sum_{p} \frac{\psi_{p}(\mathbf{r}) \psi_{p}^{*}\left(\mathbf{r}^{\prime}\right)}{\omega^{2}-k_{p}^{2}}
$$

Carrying out the volume integration,

$$
\int(d \mathbf{r}) \mathcal{G}_{\omega}(\mathbf{r}, \mathbf{r})=\sum_{p} \frac{1}{\omega^{2}-k_{p}^{2}}
$$

we find that the energy can be written in the form

$$
E=-i \int_{-\infty}^{\infty} \frac{d \omega}{2 \pi} e^{-i \omega \tau} \frac{\omega}{2} \sum_{p}\left(\frac{1}{\omega-k_{p}}+\frac{1}{\omega+k_{p}}\right) .
$$

Here we have retained a time splitting, $\tau=t-t^{\prime} \rightarrow 0$, which is a technique to regulate the divergent expression. What does this integral mean? Since the energy must be real, when $\tau$ is set equal to zero, the $-i$ is to be interpreted as an instruction to pick out the negative 
imaginary part of the integral. That means that the contour of integration $C$ must encircle all the poles on the real axis, the positive poles by a contour closed in the counterclockwise sense, and the negative poles by a contour closed in the clockwise sense: (see Ref. [31])

$$
E=-\frac{i}{8 \pi} \int_{C} \omega d_{\omega} \ln \prod_{p}\left(\omega-k_{p}\right)\left(\omega+k_{p}\right)
$$

(We may verify the sign by noting that Eq. (2.1) is formally reproduced.) This is the content of (2.7).

\section{APPENDIX B: VAN DER WAALS ENERGY OF A DIELECTRIC CYLINDER}

It is now established that for tenuous media the Casimir effect and the sum of molecular van der Waals forces are identical [29]. Here we calculate the latter for a dilute solid cylinder, with dielectric constant $\varepsilon \neq 1$ in the interior, $\varepsilon=1$ in the exterior, and $\mu=1$ everywhere. We follow the procedure given in Ref. [13]. The van der Waals energy for this sphere is

$$
E_{\mathrm{vdW}}=-\frac{1}{2} B N^{2} \int d^{D} r d^{D} r^{\prime}\left[\left|\mathbf{r}_{\perp}-\mathbf{r}_{\perp}^{\prime}\right|^{2}+r^{2}+r^{2}-2 r r^{\prime} \cos \theta\right]^{-\gamma / 2},
$$

where $B=(23 / 4 \pi) \alpha^{2}, \alpha=(\varepsilon-1) / 4 \pi N$ being the molecular polarizability, and $N$ being the number density of molecules. We have regulated the integral by dimensional continuation, $D$ being the number of spatial dimensions, and $\gamma$ being the (inverse) power of the CasimirPolder potential. The following calculation is valid providing $D>\gamma$; the final result will be obtained by violating this condition, by setting $D=3$ and $\gamma=7$.

We assume translational invariance in the $D-2$ transverse directions, so the transverse integral is easy: ( $L$ is the length of the cylinder and $b^{2}=r^{2}+r^{2}-2 r r^{\prime} \cos \theta$ )

$$
\begin{aligned}
\int_{-\infty}^{\infty} d^{D-2} r_{\perp} d^{D-2} r_{\perp}^{\prime}\left[\left|\mathbf{r}_{\perp}-\mathbf{r}_{\perp}^{\prime}\right|^{2}+b^{2}\right]^{-\gamma / 2} & =L^{D-2} \int_{-\infty}^{\infty} d^{D-2} r_{\perp}\left[r_{\perp}^{2}+b^{2}\right]^{-\gamma / 2} \\
& =\frac{L^{D-2}}{\Gamma(\gamma / 2)} \int_{-\infty}^{\infty} d^{D-2} r_{\perp} \int_{0}^{\infty} \frac{d t}{t} t^{\gamma / 2} e^{-t\left(r_{\perp}^{2}+b^{2}\right)} \\
& =\frac{L^{D-2}}{\Gamma(\gamma / 2)} \int_{0}^{\infty} d t t^{\gamma / 2-1} e^{-t b^{2}}\left[\int_{-\infty}^{\infty} d x e^{-t x^{2}}\right]^{D-2} \\
& =(L \sqrt{\pi})^{D-2}\left(b^{2}\right)^{D / 2-\gamma / 2-1} \frac{\Gamma(\gamma / 2-D / 2+1)}{\Gamma(\gamma / 2)} .
\end{aligned}
$$

The remaining integral over $r, r^{\prime}, \theta, \theta^{\prime}$ is just that given in Ref. [13. In Eq. (3.25) there, we merely set $D=2$ and $\gamma=\gamma-D+2$. The result is

$$
E_{\mathrm{vdW}}=-B N^{2} \frac{L^{D-2}}{a^{\gamma-D-2}} \frac{2^{D-\gamma} \pi^{D / 2+1 / 2} \Gamma(\gamma / 2-D / 2+1) \Gamma(D / 2-\gamma / 2+1 / 2)}{\Gamma(\gamma / 2) \Gamma(D / 2-\gamma / 2+2)(D-\gamma)} .
$$

This is exactly the result found by Romeo [32]. Now when we set $D=3$ and $\gamma=7$ everything is finite except for the second gamma function in the denominator, which has a simple pole, and thus the Casimir energy vanishes in this case. 


\section{APPENDIX C: INFINITELY THIN PERFECTLY CONDUCTING CYLINDRICAL SHELL}

We show here that the Casimir energy for an infinitely thin perfectly conducting cylindrical shell is given by Eqs. (3.18) and (3.19) with $\xi^{2}=1$. In this case, as for a perfectly conducting spherical shell, the frequencies of electromagnetic oscillations inside and outside the shell turn out to be different [16]. The frequencies of the TE-modes are determined by

$$
\begin{aligned}
J_{n}^{\prime}(\lambda a) & =0, \quad r<a, \\
H_{n}^{\prime}(\lambda a) & =0, \quad r>a .
\end{aligned}
$$

For the TM-modes we have

$$
\begin{aligned}
J_{n}(\lambda a) & =0, \quad r<a, \\
H_{n}(\lambda a) & =0, \quad r>a .
\end{aligned}
$$

In these equations

$$
\lambda^{2}=\omega^{2}-k_{z}^{2}, \quad n=0, \pm 1, \pm 2, \ldots
$$

Substituting $\Delta_{n}^{\mathrm{TE}}$ and $\Delta_{n}^{\mathrm{TE}}$ in Eq. (3.9) by the new equations (C1)-(C4) we obtain [cf. with Eq. (3.15)]

$$
E=\frac{1}{4 \pi a^{2}} \sum_{n=-\infty}^{\infty} \int_{0}^{\infty} y d y \ln \left\{-4 y^{2} I_{n}(y) I_{n}^{\prime}(y) K_{n}(y) K_{n}^{\prime}(y)\right\}
$$

To rearrange the argument of the logarithmic function in this formula we again use equalities (3.16) and (3.17). This gives Eqs. (3.18) and (3.19) with $\xi^{2}=1$ and $c=1$ :

$$
E=\frac{1}{4 \pi a^{2}} \sum_{n=-\infty}^{\infty} \int_{0}^{\infty} y d y \ln \left\{1-\left[y\left(I_{n}(y) K_{n}(y)\right)^{\prime}\right]^{2}\right\}
$$

which is, of course, the unregulated version of the result derived rigorously in Ref. [2].

\footnotetext{
${ }^{4}$ The exterior modes can be considered only formally, since the Hankel functions have only a finite number of complex zeroes. See Ref. [6]. Nevertheless, this formal procedure yields the correct result. The mode sum breaks down in this case, because the singularity structure is not that assumed in (2.7), but the Green's function method retains meaning.
} 


\section{REFERENCES}

[1] R. Balian and B. Duplantier, Ann. Phys. (N.Y.) 112, 165 (1978).

[2] L. L. DeRaad, Jr. and K. Milton, Ann. Phys. (N.Y.) 136, 229 (1981).

[3] V. V. Nesterenko and I. G. Pirozhenko, Phys. Rev. D 57, 1284 (1998).

[4] I. Brevik, V. V. Nesterenko, and I. G. Pirozhenko, Direct mode summation for the Casimir energy of a solid ball, Report No. JINR E2-97-307, Dubna, 1997, hepth/9710101; to be published in J. Phys. A: Math. Gen.

[5] V. V. Nesterenko and I. G. Pirozhenko, J. Math. Phys. 38, 6265 (1997).

[6] M. E. Bowers and C. R. Hagen, "Casimir Energy of a Spherical Shell,' preprint UR-1533.

[7] I. Brevik and G. H. Nyland, Ann. Phys. (N.Y.) 230, 321 (1994).

[8] L. L. DeRaad, Jr., Fortschr. Phys. 33, 117 (1985).

[9] K. A Milton, L. L. DeRaad Jr., and J. Schwinger, Ann. Phys. (N.Y.) 115, 338 (1978).

[10] K. A Milton, Ann. Phys. (N.Y.) 127, 49 (1980).

[11] K. A. Milton, in Proceedings of the Third Workshop on Quantum Field Theory Under the Influence of External Conditions, Leipzig, 1995, edited by M. Bordag, (Teubner, Stuttgart, 1996), p. 13.

[12] K. A. Milton and Y. J. Ng, Phys. Rev. E 55, 4207 (1997).

[13] K. A. Milton and Y. J. Ng, Phys. Rev. E 57, 5504 (1998).

[14] I. Brevik and H. Kolbenstvedt, Ann. Phys. (N.Y.) 143, 179 (1982); 149, 237 (1983).

[15] I. Brevik, J. Phys. A: Math. Gen. 20, 5189 (1987).

[16] J. A. Stratton, Electromagnetic Theory (McGraw-Hill, New York, 1941).

[17] In Ref. [16, Section 9.15] Eq. (2.3) is considered in connection with the propagation of electromagnetic waves of frequency $\omega$ along a solid cylinder. As a consequence it is treated there as an equation determining the wave number $k_{z}$ at a given $\omega$.

[18] T. D. Lee, Particle Physics and Introduction to Field Theory (Harwood, New York, 1981).

[19] I. Brevik, J. Phys. A: Math. Gen. 15, L369 (1982); Can. J. Phys. 61, 493 (1983).

[20] I. Brevik and H. Kolbenstvedt, Can. J. Phys. 62, 805 (1984); 63, 1409 (1985); Phys. Rev. D 25, 1731 (1982).

[21] Louis De Broglie, Problems de propagations guidees des ondes electromagnetiques (Gauthiers-Villars, Paris, 1941).

[22] J. Schwinger, L. L. DeRaad, Jr., K. A. Milton, and W.-y. Tsai, Classical Electrodynamics (Perseus, Reading, Massachusetts, 1998).

[23] M. Abramowitz and I. A. Stegun, Handbook of Mathematical Functions (National Bureau of Standards, Washington, D. C., 1964; reprinted by Dover, New York, 1972).

[24] E. Elizalde, S. D. Odintsov, A. Romeo, A. A. Bytsenko, and S. Zerlini, Zeta regularization techniques with applications (World Scientific, Singapore, 1994).

[25] E. Elizalde, Ten Physical Applications of Spectral Zeta Functions (Springer, Berlin, 1995).

[26] Applying the Green's function techniques this problem has been considered in [7].

[27] P. Gosdzinsky and A. Romeo, Energy of the vacuum with a perfectly conducting and infinite cylindrical surface, hep-th/9809199.

[28] C. M. Bender and K. A. Milton, Phys. Rev. D 50, 6547 (1994); K. A. Milton, ibid. 55, 4940 (1997). 
[29] I. Brevik, V. N. Marachevsky, and K. A. Milton, "Identity of the van der Waals and the Casimir Effect and the Irrelevance of these Phenomenon to Sonoluminescence," OKHEP-98-08.

[30] G. Barton, "Perturbative Check on the Casimir Energy of a Nondispersive Dielectric Ball."

[31] R. Kantowski and K. A. Milton, Phys. Rev. D 35, 549 (1987).

[32] A. Romeo, private communication. 